\title{
Coeficiente emocional en niños y adolescentes de Boyacá, Colombia. Estudio comparativo
}

Rafael Enrique Buitrago Bonilla

Doctor en Educación Musical

Universidad Pedagógica y Tecnológica de

Colombia

lafaremus@gmail.com

https://orcid.org/o000-0001-7553-6473

\section{Lucía Herrera Torres}

Doctora en Psicología

Universidad de Granada - España

luciah@ugr.es

http://orcid.org/oooo-0002-5860-1357

Ruth Nayibe Cárdenas Soler

Doctora en Educación Musical

Universidad Pedagógica y Tecnológica de

Colombia

ruth.cardenas@uptc.edu.co

https://orcid.org/0000-0003-4997-4116

Artículo de investigación

Recepción: 29 de abril de 2019 Aprobación: 30 de julio de 2019

https://doi.org/10.19053/22160159.v10.n25.2019.10002

\section{Resumen}

Este artículo analiza comparativamente la inteligencia emocional de niños y jóvenes estudiantes colombianos de dos rangos de edad, 8-10 y 11-16 años. La muestra del estudio fue de 1451 estudiantes de tres provincias del departamento de Boyacá. La investigación se realizó desde una perspectiva cuantitativa. Para la recolección de información se empleó el Inventario de Coeficiente Emocional: versión para jóvenes [EQ-i: YV]. Tanto la fiabilidad como la validez del EQ-i: YV fueron determinadas para esta muestra. Los principales resultados mostraron algunas diferencias en la inteligencia emocional según el rango de edad. Los niños obtuvieron mejores resultados que los adolescentes en las escalas de manejo del estrés y adaptabilidad.

Palabras clave: coeficiente emocional, inteligencia emocional y social, niños, adolescentes, estudiantes 


\title{
Emotional quotient in children and adolescents from Boyacá, Colombia: a comparative study
}

\begin{abstract}
This article makes a comparative analysis of emotional intelligence of Colombian children and young students aged from 8 to 10 year-old and from 11 to 16 years-old. The study sample was 1451 students from three provinces in the Department of Boyacá. The research was carried out from a quantitative perspective. Emotional Quotient Inventory: Youth Version [EQ-i: YV] was used for data collection. Both the reliability and the validity of the EQ-i: YV were determined for the sample. The main results showed some differences in emotional intelligence according to age range. Children achieved better results than adolescents in stress management and adaptability scales.
\end{abstract}

Keywords: emotional quotient, social and emotional intelligence, children, adolescents, students

\section{Le quotient émotionnel chez des enfants et des adolescents de Boyacá, en Colombie : une étude comparative}

\section{Résumé}

Cet article présente une analyse comparative de l'intelligence émotionnelle des enfants et de jeunes élèves colombiens appartenant à deux groupes d'âge : de 8 à 10 ans et de 11 à 16 ans. L'échantillon de cette étude a été constitué par 1451 élèves de trois provinces du département de Boyacá. La recherche a été abordée d'un point de vue quantitatif. L'inventaire du quotient émotionnel : 
version pour les jeunes [EQ-i: YV] a été utilisé pour la collecte des données. Tant la fiabilité que la validité de l'EQ-i: YV ont été déterminées pour l'échantillon. Les principaux résultats ont montré certaines différences par rapport à l'intelligence émotionnelle en fonction des groupes d'âge. Les enfants ont enregistré de meilleurs résultats que les adolescents dans les barèmes de gestion du stress et d'adaptabilité.

Mots-clés : quotient émotionnel, intelligence émotionnelle et sociale, enfants, adolescents, élèves

\section{Coeficiente emocional em crianças e adolescentes de Boyacá, na Colômbia. Estudo comparativo}

\section{Resumo}

Este artigo analisa comparativamente a inteligência emocional de crianças e jovens estudantes colombianos de duas faixas etárias, 8-10 e 11-16 anos. A mostra do estudo foi de 1451 estudantes de três províncias do departamento de Boyacá. A pesquisa foi realizada desde uma perspectiva quantitativa. Para a coleta de informação, empregou-se o Inventário de Coeficiente Emocional: versão para jovens [EQ-i: YV]. Tanto a confiabilidade como a validade do EQ-i: YV foram determinadas para esta mostra. Os principais resultados mostraram algumas diferenças na inteligência emocional segundo a faixa etária. Os meninos obtiveram melhores resultados que os adolescentes nas escalas de manejo do estresse e adaptabilidade.

Palavras-chave: coeficiente emocional, inteligência emocional e social, crianças, adolescentes, estudantes 


\section{Introducción}

\section{La inteligencia emocional y social en la escuela.}

La inteligencia emocional y social está relacionada con el desarrollo y desempeño humanos y el éxito en la vida. Se relaciona de manera directa con la toma de decisiones, lo que la hace diferente - pero complementaria - a la inteligencia cognitiva (BarOn, Tranel, Denburg \& Bechara, 2003). Además, las emociones son determinantes en las conductas personales e interpersonales, es decir, afectan los vínculos y relaciones de las personas (FernándezBerrocal \& Extremera, 2006; Herrera, Buitrago \& Cepero, 2017; Herrera, Buitrago, Lorenzo \& Badea, 2015).

En los últimos años se ha ratificado que las emociones y el bienestar son fundamentales para el desarrollo tanto personal como académico de los estudiantes. En esta dirección, se ha evidenciado que el desarrollo de las habilidades emocionales contribuye al rendimiento académico e incide en la disminución de la ansiedad. No obstante, estos resultados son más relevantes en la educación primaria que en la educación secundaria (Cabello, Pérez, Ros \& Filella, 2019).

De igual manera, la validez predictiva de la inteligencia emocional [IE] se ha evidenciado en algunas variables del contexto escolar como el acoso, la salud mental y el bienestar (Droppert et al., 2019). Igualmente, se ha establecido la relación entre la IE y la resiliencia (Armstrong, Galligan \& Critchley, 2011; Connor \& Slear, 2009; Magnano, Craparo \& Paolillo, 2016). Asimismo, el reconocimiento y la gestión emocional incrementan la sensación de confianza, comodidad, apoyo, aceptación de otras personas, optimismo y adaptabilidad, lo que implica vínculos de apoyo con pares, maestros y familiares, aspecto que incide en la generación de redes de apoyo (Ciarrochi, Chan \& Bajgar, 2001; Gumora \& Arsenio, 2002). No obstante, desde la perspectiva de Droppert et al. (2019), aún no son claros los motivos que generan la relación entre IE y rendimiento escolar.

La regulación emocional y, en especial, el modelo de Gross (1998) se ha utilizado en estudios de carácter clínico (Aldao, NolenHoeksema \& Schweizer, 2010; Kring \& Sloan, 2010) y no clínico (Llewellyn, Dolcos, Iordan, Rudolph \& Dolcos, 2013; Webb, Miles \& Sheeran, 2012). Sin embargo, los estudios que analizan la relación 
entre regulación emocional y el bienestar en la adolescencia son pocos (Pastor, López-Penadés, Cifre \& Moliner-Urdiales, 2019). En esta dirección, desde la perspectiva de Wang, Wei, Yi, Lo y Lee (2019), la investigación respecto a la regulación de las emociones de niños y adolescentes en Asia Oriental es escasa debido, en gran medida, a la inexistencia de escalas de medición con validez cultural, motivo por el cual es imprescindible señalar que los estudios sobre la regulación de las emociones requieren integrar los fundamentos teóricos, el contexto cultural y las experiencias prácticas.

Desde la perspectiva de la regulación emocional, la cual puede llevarse a cabo de manera consciente o inconsciente, Gross (1998, 2015) plantea un modelo que integra cinco estrategias para alcanzar dicha regulación: elección de la situación, modificación de la situación, despliegue de la atención, cambio cognitivo y modulación de la respuesta. Estas estrategias se orientan hacia el antecedente o la respuesta (Pastor et al., 2019).

Lo cierto es que la regulación emocional tiene una importante incidencia en los ajustes psicológicos y sociales, así como en el bienestar. En oposición, los inconvenientes para regularlas se relacionan con problemas de comportamiento, trastornos de internalización, conflictos interpersonales, menor tolerancia a eventos estresantes y a la ansiedad (Aldao \& Christensen, 2015; Eastabrook, Flying \& Hollestein, 2014; Gullone, Hughes, King \& Tonge, 2010; Gullone \& Taffe, 2012; Hu et al., 2014). En esta dirección, Schoeps, Montoya-Castilla y Raufelder (2019) advierten que los beneficios y el efecto protector de la IE pueden llegar a disminuir por el estrés percibido, en cuanto a la satisfacción con la vida.

Son múltiples los programas de intervención socioemocional que se han creado e implementado y que han evidenciado resultados positivos, en particular en lo referente al rendimiento académico, el bienestar y la salud mental (Blair, McKinnon \& Daneri, 2018; Coelho, Marchante \& Sousa, 2015; Meyers, Domitrovich, Dissi, Trejo \& Greenberg, 2019; Panayiotou, Humphrey \& Wigelsworth, 2019). A ello se suma el vínculo positivo de los estudiantes con la escuela, el cual deriva, en gran medida, de los ambientes de aprendizaje positivos y los vínculos fundamentados en la confianza (Agulló, Filella, Soldevila \& Ribes, 2011; Brackett, Mayer \& Warner, 2004; Cherniss, Extein, Goleman \& Weissberg, 2006; Eren, Ergun 
\& Altintas, 2009; Gil-Olarte, Palomera \& Brackett, 2006; Greenberg et al., 2003).

De la misma manera, son múltiples las investigaciones que han relacionado la IE y el contexto escolar. Así, por ejemplo, se han evidenciado hallazgos de la asociación entre los hábitos de lectura, el desarrollo de competencias lectoras y la IE (Jiménez, Alarcón \& Vicente-Yague, 2019); la regulación emocional y el desarrollo social (Brackett, Rivers, Shiffman, Lerner \& Salovey, 2006; Charbonneau \& Nicol, 2002; Engelberg \& Sjöberg, 2004; Lopes et al., 2004; Lopes, Salovey, Beers \& Coté, 2005; Mayer, Roberts \& Barsade, 2008; Mestre, Guil, Lopes, Salovey \& Gil-Olarte, 2006; Reis et al., 2007; Zavala, Valadez \& Vargas, 2008); el absentismo escolar y los problemas de comportamiento (Petrides, Frederickson \& Furnham, 2004); la relación de la autoestima y el desempeño en los grupos (Barling, Slater, \& Kelloway, 2000; Gil-Olarte et al., 2006; Palmer, Walls, Burgess \& Stough, 2001); así como la adaptación al contexto escolar (Extremera \& Fernández-Berrocal, 2003, 2004), entre otros aspectos.

\section{La inteligencia emocional y social en los niños.}

La alfabetización emocional implica reconocer y poner en palabras las emociones, lo que requiere una participación fundamental de los padres, e involucra procesos reflexivos y de discusión respecto a las emociones. En este sentido, cuando los niños no han podido experimentar en su entorno vínculos y relaciones que propicien la expresión de emociones y sentimientos, incluyendo la participación del lenguaje no verbal, suelen manifestar dificultades para entender las emociones e intenciones de otras personas (Dayton, 2009). Esta disfunción suele incluir, además de la imprecisión de la expresión emocional en una situación específica, la imposibilidad para controlar las emociones, lo cual suele generar relaciones y vínculos inadecuados (Barrio, 2005).

En niños de 6-10 años se ha evidenciado que cuando se generan cambios dinámicos en el lóbulo prefrontal se presenta una tendencia a percibir emociones empáticas. En esta dirección se puede señalar que la empatía está relacionada con las representaciones interiores de los estados emocionales, tanto propios como de los demás. Por esta razón, se presenta una relación con la actividad asimétrica del córtex dorsolateral y del área frontopolar (Light et al., 2009). 
Lo cierto es que las investigaciones han permitido establecer que los niños que logran reconocer sus emociones presentan menos posibilidades de experimentar ansiedad y agresividad cuando van a la escuela. Por el contrario, aquellos que han tenido experiencias de maltrato, como es predecible, se mantienen a la defensiva y suelen reaccionar con facilidad. Por esta razón, es de gran relevancia el periodo de los tres a los nueve años de edad, ya que durante esta etapa, al aprender a designar las emociones, se estimulan conexiones sinápticas que vinculan al hipocampo, en especial la amígdala con el lóbulo frontal. De este modo, durante dicha etapa es muy fácil el desarrollo de habilidades emocionales (Greenberg, 2003).

Es evidente que el desarrollo emocional y la participación familiar inciden en el aprendizaje de los alumnos, motivo por el cual se pueden considerar como predictores. En esta dirección, en una investigación realizada con un grupo de estudiantes chilenos de 7-11 años, se puso de manifiesto que el impacto de la participación familiar en el aprendizaje no está mediado por el desarrollo emocional (Saracostti et al., 2019). Ahora bien, se han encontrado hallazgos respecto a la importancia de programas de intervención en IE orientados a estudiantes "en riesgo" para prevenir conductas de internalización y problemas de depresión y soledad sostenida en la infancia media (Davis, Nowland \& Qualter, 2019).

Para Shapiro (2008), los niños cuentan con la capacidad cerebral para comprender y expresar emociones y sentimientos, y también con la disposición para hacerlo, pero esta última está determinada por el contexto cultural y por los vínculos y relaciones con pares, padres, maestros y adultos en general. Es por ello que los vínculos y experiencias del niño a partir de sus relaciones interpersonales son decisivas en su futuro emocional y social (Greenberg, 2003). Por lo tanto, el proceso de alfabetización emocional, así como del desarrollo de habilidades socioemocionales, requiere que se continúe y potencie en la escuela de la mano de los maestros (Bisquerra, 2009; Buitrago \& Herrera, 2013; Fernández-Berrocal \& Extremera, 2006; Palomera, Fernández-Berrocal \& Brackett, 2008), proceso en el cual es fundamental tener en cuenta la relación entre los sentidos atribuidos a las emociones y la identidad profesional docente (Buitrago, Ávila \& Cárdenas, 2017; Buitrago \& Cárdenas, 2017), así como el desarrollo de la empatía (Gerdes \& Segal, 2011; Gerdes, Segal, Jackson \& Mullins, 2011; Herrera, Buitrago \& Ávila, 2016). 


\section{La inteligencia emocional y social en la adolescencia temprana.}

El periodo crítico de la adolescencia en general, pero de manera particular de la adolescencia temprana, está marcado por importantes cambios biológicos y una actividad hormonal intensa (Zimmerman \& Iwanski, 2014), motivo por el cual se suelen presentar una importante cantidad de conflictos con los padres y adultos, así como una relevante presencia y despliegue de emociones con tendencia negativa, lo que implica grandes desafíos para la regulación emocional (Pastor et al., 2019). Es por ello que la gestión emocional contribuye de manera positiva a la reducción del estrés en el rendimiento escolar (Boekaerts, 1993; Pressman \& Cohen, 2005). Desde esta perspectiva, se puede señalar que los adolescentes que se enfadan con mayor facilidad suelen cometer más errores y presentar un menor rendimiento escolar. Esto es debido, en gran medida, a que demoran más tiempo en recuperarse de dicho estado emocional. En cualquier caso, muchos de los problemas emocionales que experimentan los adolescentes son diferentes a los que tienen lugar en los niños en edad escolar (Droppert et al., 2019). Todo esto obedece a los múltiples cambios neurológicos, físicos y emocionales que se generan durante esta etapa (Greenberg \& Snell, 1997).

No obstante, Chen (2019) establece que en el contexto escolar de los adolescentes chinos el apoyo de los amigos es determinante para configurar la relación entre la IE y la resiliencia. Sin embargo, el apoyo de la familia en este sentido no es significativo. En cualquier caso, se establece que el tipo de escuela es determinante en este tipo de relaciones. Lo cierto es que la habilidad para gestionar las emociones disminuye la probabilidad de abuso en el consumo de alcohol en los adolescentes (González-Yubero, Palomera \& LázaroVisa, 2019).

De igual manera, se ha encontrado disparidad interpersonal en lo referente a las maneras y niveles de regulación emocional, los cuales inciden en el pensamiento y la conducta (Mozaz, Mestre \& Núñez-Vázquez, 2009). Desde una perspectiva evolutiva, son múltiples las competencias que se desarrollan durante varias etapas y que de alguna manera tienen un momento relevante al llegar a la adolescencia, como la autoconciencia y la regulación emocional, la empatía, la denominación de las propias emociones, la relación entre las expresiones con los estados emocionales internos, el 
manejo del estrés, las relaciones y vínculos interpersonales y la eficacia emocional (Saarni, 2000).

En el contexto escolar, el aprendizaje y diferentes aspectos, como la motivación, adquieren relevancia y se convierten en objeto de estudio tanto en niños como en adolescentes (Roeser, Van der Wolf \& Strobel, 2001). En esta dirección, la adolescencia es fundamental para el desarrollo de habilidades mentales y emocionales, debido a que durante esta etapa se generan interrogantes existenciales que luego desaparecen y solo se vuelven a presentar en la vejez (DalaiLama \& Ekman, 2009). Desde otra perspectiva, Babić y Buško (2015), al analizar el género y la edad en relación con la IE y el rendimiento académico en la adolescencia temprana, encontraron diferencias en cuanto al género pero no en cuanto a la edad.

Modelo de inteligencia social y emocional (Bar-On, 1997, 2006).

La Emotional-Social Intelligence [ESI] es un modelo que integra las habilidades tanto emocionales como sociales, motivo por el cual enfatiza en el ámbito intrapersonal e interpersonal, al integrar aspectos de la configuración y el funcionamiento social y emocional para potenciar el bienestar psicológico, a partir de diferentes factores no cognitivos (Bar-On, 2000, 2006). El modelo se configura a partir de cinco ámbitos: intrapersonal, interpersonal, adaptabilidad, manejo del estrés y estado general de ánimo, los cuales, a su vez, integran 15 aspectos (figura 1). El modelo permite a partir de estos ámbitos establecer el coeficiente emocional.

Modelo de Inteligencia Social y Emocional (Bar-On, 1997)

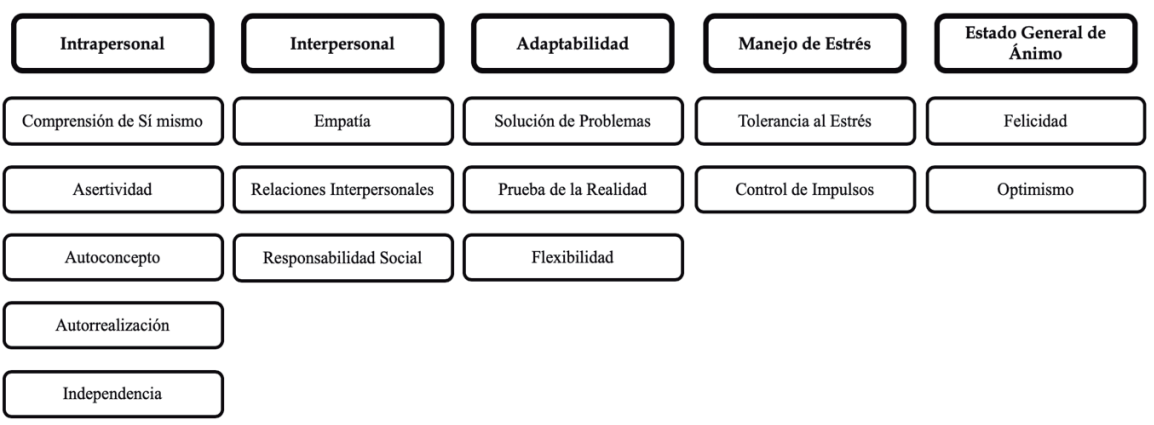

Figura 1. Modelo mixto de inteligencia social y emocional de Bar-On (1997). Nota: Este modelo se puede consultar en Buitrago (2012) y, Buitrago y Herrera (2015). 
Por consiguiente, la edad tiene una incidencia fundamental en el desarrollo y comprensión de las emociones, en gran medida, porque el pensamiento analítico posibilita una mayor consciencia de las experiencias emocionales, así como la profundidad en cuanto a las representaciones y el entendimiento de las mismas (Albanese, Stasio, Chiacchio, Fiorilli \& Pons, 2010). En consecuencia, el reconocimiento de las emociones mejora con los años y está asociado con la calidad de las amistades, el afecto positivo, la satisfacción con la vida, los vínculos interpersonales, el desarrollo social, la satisfacción con la vida, la percepción del apoyo social y el afecto positivo (Mitrofan \& Ciuluvică, 2012; Verzeletti, Zammuner, Galli \& Agnoli, 2016; Wang, Hawk, Tang, Schlegel \& Zou, 2019). Además de ello, Wang et al. (2019) enfatizan en que suelen ser las niñas quienes presentan mejores habilidades en cuanto al reconocimiento emocional.

En consecuencia, el presente estudio analiza comparativamente el coeficiente emocional de niños $-8-10$ años- y adolescentes tempranos -11-16 años-, desde el modelo de inteligencia social y emocional (Bar-On, 1997, 2006), en tres provincias del departamento de Boyacá en Colombia.

\section{Método}

La presente investigación se realizó desde una perspectiva cuantitativa. Se utilizó un tipo de muestreo aleatorio por conglomerados, constituyendo cada conglomerado cada uno de los centros o instituciones educativas de las provincias Centro, Tundama y Sugamuxi del departamento de Boyacá en Colombia.

\section{Participantes}

El estudio se realizó con una muestra inicial de 1876 estudiantes, de la cual se eliminaron los casos inválidos, haciendo uso del índice de inconsistencia del Bar On EQ-i: YV, por lo que la muestra final fue de 1451 estudiantes: 727 niñas $-50,1 \%-$ y 724 niños -49 , $9 \%-$, con un rango de edad de 8 a 16 años y una edad promedio de 10,38 años $(D T=1,12)$. Los participantes eran estudiantes tanto de instituciones educativas rurales $(n=690)-47,6 \%-$, como urbanas, $(n=761)-52,4 \%-$. Además, los participantes vivían en una de tres provincias del departamento de Boyacá en Colombia: Centro $(n=$ 
478) $-32,9 \%-$, Tundama $(n=483)-33,3 \%-$ y Sugamuxi $(n=$ 490) $-33,8 \%$ -

La distribución de la muestra, según su ubicación y género, consistió en 344 niñas en las áreas rurales -49,85\%- y 346 niños $-50,15 \%$ - Las áreas urbanas incluyeron 383 niñas -50,32\%y 378 niños $-49,68 \%$-. Es relevante señalar que la proporción de estudiantes de áreas rurales y urbanas en cada una de las tres provincias fue muy cercana. Así, en la provincia Centro, el 49,6\% vivía en una zona rural ( $n=237)$ y el $50,4 \%$ en una zona urbana $(n=241)$; en la provincia de Tundama, el $45.8 \%$ vivía en una zona rural $(n=221)$ y el $54,2 \%$ en una zona urbana $(n=262)$; y en la provincia de Sugamuxi, el $47,3 \%$ vivía en una zona rural $(n=232)$ y el $52,7 \%$ en una zona urbana $(n=258)$.

\section{Instrumento}

Para el estudio se utilizó el Inventario de Coeficiente Emocional: Versión Juvenil [EQ-i: YV] (Bar-On \& Parker, 2000), el cual es un instrumento de autoinforme diseñado para medir la IE en niños y jóvenes. Consta de 60 ítems y se compone de siete escalas o dominios: intrapersonal -6 ítems-; interpersonal -12 ítems-; manejo del estrés -12 ítems-; y adaptabilidad, -10 ítems-, estas cuatro escalas generan el coeficiente emocional total -EQ total-. A ellas se suman el estado general de ánimo -14 ítems-, el cual se considera un facilitador del coeficiente emocional, aunque no integra su valor, y la escala de impresión positiva -6 ítems-, que permite evidenciar cuando una persona cuenta con una exagerada percepción positiva de sí misma. Por último, el cuestionario cuenta con un índice de inconsistencia, el cual es un indicador de las respuestas al azar.

El BarOn EQ-i: YV está diseñado para ser empleado con niños y adolescentes dentro del rango de edad de 7 a 18 años. Se responde según una escala tipo Likert de cuatro puntos $-1=$ No es verdad en mi caso, 4= Muy cierto en mi caso- . La corrección de las pruebas se realiza por género y grupo de edad -7-9, 10-12, 13-15 y 16-18 años-. Los puntajes permiten obtener tres medidas: puntajes estándar, nivel -marcadamente bajo, muy bajo, bajo, promedio, alto, muy alto y marcado alto-y percentiles. Además de estimar el nivel de IE, genera un perfil emocional y social. 
Bar-Ony Parker (2000) establecieron los criterios psicométricos con respecto a la validez y fiabilidad de la prueba. Además, estos se han confirmado en diferentes poblaciones, como la húngara (Kun et al., 2012) y la española (Ferrándiz, Hernández, Bermejo, Ferrando \& Sáinz, 2012). Para el presente estudio, se realizó un análisis factorial. Se ha verificado la idoneidad de la prueba, mediante el índice Kaiser-Meyer-Olkin $(K M O=0,724)$ y la prueba de esfericidad de Bartlett ( $\left.\chi^{2}=35,932, p=0,000\right)$. Posteriormente, se desarrolló un análisis factorial exploratorio. Se utilizó la extracción de componentes principales con rotación Varimax, que explica el $45,629 \%$ de la varianza total. Se obtuvo la siguiente distribución de factores: estado de ánimo general $-11,960 \%-$, manejo del estrés $-9,624 \%-$, adaptabilidad -9,018\%-, interpersonal -8,802\%-e intrapersonal $-6,225 \%-$. De manera similar, la fiabilidad general de la prueba obtuvo un alfa de Cronbach de 0,802 y para cada dimensión: intrapersonal $(\alpha=0,634)$, interpersonal $(\alpha=0,751)$, manejo del estrés $(\alpha=0,786)$, adaptabilidad $(\alpha=0,727)$ y estado de ánimo general $(\alpha=0,813)$.

\section{Procedimiento}

Se obtuvo el aval y aprobación del secretario de educación del departamento de Boyacá y de los secretarios de educación de los municipios certificados de las tres provincias participantes. Luego, se gestionó el aval y apoyo de los rectores de cada una de las instituciones educativas participantes y se aplicaron los cuestionarios en el aula regular durante alguna de las jornadas escolares. Los participantes contestaron al cuestionario después de haber recibido las instrucciones correspondientes para ello.

Una vez que se obtuvo la totalidad de los datos, se eliminaron los casos no válidos, aquellos que obtuvieron un valor igual o superior a 10 en el índice de inconsistencia del Bar-On EQ-i: YV, $n=425$. En esta dirección, es importante señalar que no se presentaron casos con una puntuación estándar en la impresión positiva de dimensión igual o superior a 130, Mínimo = 63, Máximo $=118, M=90,69, D T=11.46$.

En cuanto al análisis de datos, se utilizó como apoyo el paquete estadístico SPSS para Windows (versión 22.0). Se hallaron los estadísticos descriptivos -media, desviación típica, frecuencias y porcentajes-. Se realizó un análisis de varianza en función de la variable rango de edad para los percentiles del BarOn EQ-i: YV 
según el rango de edad. Para las comparaciones post-hoc se utilizó el estadístico Bonferroni. Además, se empleó la prueba $C h i^{2}$ para determinar si existían diferencias en los diferentes niveles de cada una de las escalas del BarOn EQ-i: YV.

\section{Resultados}

Para analizar si existían diferencias en los percentiles de las escalas del BarOn EQ-i: YV, en función de los rangos de edad, se realizó un análisis de varianza (tabla 1). Los resultados mostraron diferencias estadísticamente significativas en las escalas de manejo del estrés $\left(F_{(1,1449)}=6,197, p<0,05, E t a^{2}=0,004\right)$ y adaptabilidad $\left(F_{(1,1449)}=3,903, p<0,05, E t a^{2}=0,003\right)$.

\section{Tabla 1}

Estadísticos descriptivos, por rango de edad, de los percentiles de las escalas del BarOn EQ-i: YVy resultados del Anova

\begin{tabular}{|c|c|c|c|c|c|c|}
\hline EQi: YV & Rango edad & Media & $D T$ & $F$ & $p$ & $E t a^{2}$ \\
\hline \multirow{3}{*}{ Intrapersonal } & 8-10 años & 53,57 & 27,62 & 3,385 & 0,066 & 0,002 \\
\hline & 11-16 años & 56,25 & 26,47 & & & \\
\hline & Total & 54,65 & 27,19 & & & \\
\hline \multirow{3}{*}{ Interpersonal } & 8-10 años & 35,98 & 26,53 & 1,683 & 0,195 & 0,001 \\
\hline & 11-16 años & 34,11 & 27,50 & & & \\
\hline & Total & 35,23 & 26,93 & & & \\
\hline \multirow{3}{*}{$\begin{array}{l}\text { Manejo del } \\
\text { estrés }\end{array}$} & 8-10 años & 46,57 & 25,25 & $6,197^{*}$ & 0,013 & 0,004 \\
\hline & 11-16 años & 43,24 & 24,71 & & & \\
\hline & Total & 45,23 & 25,08 & & & \\
\hline \multirow{3}{*}{ Adaptabilidad } & 8-10 años & 53,84 & 28,84 & $3,903^{*}$ & 0,048 & 0,003 \\
\hline & 11-16 años & 50,78 & 29,14 & & & \\
\hline & Total & 52,61 & 28,99 & & & \\
\hline \multirow{3}{*}{ EQ total } & 8-10 años & 45,23 & 25,31 & 1,649 & 0,199 & 0,001 \\
\hline & 11-16 años & 43,51 & 24,48 & & & \\
\hline & Total & 44,54 & 24,98 & & & \\
\hline \multirow{3}{*}{ Estado de ánimo } & 8-10 años & 61,78 & 26,47 & 2,274 & 0,132 & 0,002 \\
\hline & 11-16 años & 59,61 & 27,44 & & & \\
\hline & Total & 60,90 & 26,88 & & & \\
\hline \multirow{3}{*}{$\begin{array}{c}\text { Impresión } \\
\text { positiva }\end{array}$} & 8-10 años & 69,75 & 27,59 & 3,771 & 0,052 & 0,003 \\
\hline & 11-16 años & 72,54 & 25,75 & & & \\
\hline & Total & 70,87 & 26,89 & & & \\
\hline
\end{tabular}

Nota: ${ }^{*} p<0,05$ 
Posterior a ello, se realizaron comparaciones post-hoc, a través de la prueba de Bonferroni, las cuales permitieron evidenciar diferencias significativas a favor de los niños -8-10 años- frente a los adolescentes -11-16 años- en los percentiles de las escalas manejo del estrés $(t=3,335, p<0,05)$ y adaptabilidad $(t=3,062$, $p<0,05)$.

Los niveles en las escalas del BarOn EQ-i: YV para cada rango de edad así como los resultados del análisis de frecuencias se muestran en la tabla 2. Como puede observarse, las diferencias fueron estadísticamente significativas en todos los casos.

Tabla 2

Estadísticos descriptivos en los niveles de cada una de las escalas del BarOn EQ-i: YV en función del rango de edad y resultados de la prueba $\mathrm{Chi}^{2}$

\begin{tabular}{|c|c|c|c|c|c|c|c|c|c|c|}
\hline EQi:YV & $\begin{array}{l}\text { Rango } \\
\text { de edad }\end{array}$ & Marc. bajo & Muy bajo & Bajo & Medio & Alto & Muy alto & $\begin{array}{l}\text { Marc. } \\
\text { alto }\end{array}$ & Chi2 & $p$ \\
\hline \multirow{6}{*}{ Intrapersonal } & $8-10$ & 24 & 68 & 114 & 474 & 145 & 31 & 10 & $1275,693^{* * *}$ & 0,000 \\
\hline & & $2,8 \%$ & $7,9 \%$ & $13,2 \%$ & $54,7 \%$ & $16,7 \%$ & $3,6 \%$ & $1,2 \%$ & & \\
\hline & $11-16$ & 11 & 34 & 81 & 340 & 93 & 23 & 3 & $1001,966^{* k *}$ & 0,000 \\
\hline & & $1,9 \%$ & $5,8 \%$ & $13,8 \%$ & $58,1 \%$ & $15,9 \%$ & $3,9 \%$ & $0,5 \%$ & & \\
\hline & Total & 35 & 102 & 195 & 814 & 238 & 54 & 13 & $2273,227^{7 * *}$ & 0,000 \\
\hline & & $2,4 \%$ & $7,0 \%$ & $13,4 \%$ & $56,1 \%$ & $16,4 \%$ & $3,7 \%$ & $0,9 \%$ & & \\
\hline \multirow{6}{*}{ Interpersonal } & 8-10 & 57 & 117 & 195 & 410 & 77 & 10 & 57 & $721,242^{* \star *}$ & 0,000 \\
\hline & & $6,6 \%$ & $13,5 \%$ & $22,5 \%$ & $47,3 \%$ & $8,9 \%$ & $1,2 \%$ & $6,6 \%$ & & \\
\hline & 11-16 & 53 & 83 & 135 & 242 & 67 & 5 & 53 & $348,344^{* \star *}$ & 0,000 \\
\hline & & $9,1 \%$ & $14,2 \%$ & $23,1 \%$ & $41,4 \%$ & $11,5 \%$ & $0,9 \%$ & $9,1 \%$ & & \\
\hline & Total & 110 & 200 & 330 & 652 & 144 & 15 & 0 & $1059,262^{* * *}$ & 0,000 \\
\hline & & $7,6 \%$ & $13,8 \%$ & $22,7 \%$ & $44,9 \%$ & $9,9 \%$ & $1,0 \%$ & $0,0 \%$ & & \\
\hline \multirow{6}{*}{$\begin{array}{l}\text { Manejo del } \\
\text { estrés }\end{array}$} & 8-10 & 17 & 60 & 164 & 501 & 101 & 22 & 1 & $1498,092^{* * *}$ & 0,000 \\
\hline & & $2,0 \%$ & $6,9 \%$ & $18,9 \%$ & $57,9 \%$ & $11,7 \%$ & $2,5 \%$ & $0,1 \%$ & & \\
\hline & 11-16 & 6 & 56 & 121 & 332 & 58 & 12 & 0 & $764,179^{* * *}$ & 0,000 \\
\hline & & $1,0 \%$ & $9,6 \%$ & $20,7 \%$ & $56,8 \%$ & $9,9 \%$ & $2,1 \%$ & $0,0 \%$ & & \\
\hline & Total & 23 & 116 & 285 & 833 & 159 & 34 & 1 & 2483,362 & 0,000 \\
\hline & & $1,6 \%$ & $8,0 \%$ & $19,6 \%$ & $57,4 \%$ & $11,0 \%$ & $2,3 \%$ & $0,1 \%$ & & \\
\hline \multirow{6}{*}{ Adaptabilidad } & $8-10$ & 18 & 69 & 99 & 427 & 190 & 59 & 4 & $1048,185^{\star \star *}$ & 0,000 \\
\hline & & $2.1 \%$ & $8,0 \%$ & $11,4 \%$ & $49,3 \%$ & $21,9 \%$ & $6,8 \%$ & $0,5 \%$ & & \\
\hline & 11-16 & 11 & 52 & 87 & 298 & 91 & 43 & 3 & $723,306^{* * *}$ & 0,000 \\
\hline & & $1,9 \%$ & $8,9 \%$ & $14,9 \%$ & $50,9 \%$ & $15,6 \%$ & $7,4 \%$ & $0,5 \%$ & & \\
\hline & Total & 29 & 121 & 186 & 725 & 281 & 102 & 7 & $1757,697^{\star \star \star}$ & 0,000 \\
\hline & & $2,0 \%$ & $8,3 \%$ & $12,8 \%$ & $50,0 \%$ & $19,4 \%$ & $7,0 \%$ & $0,5 \%$ & & \\
\hline
\end{tabular}




\begin{tabular}{|c|c|c|c|c|c|c|c|c|c|c|}
\hline \multirow{6}{*}{ EQ total } & $8-10$ & 13 & 61 & 165 & 512 & 92 & 20 & 3 & $1576,176^{\text {*k* }}$ & 0,000 \\
\hline & & $1,5 \%$ & $7,0 \%$ & $19,1 \%$ & $59,1 \%$ & $10,6 \%$ & $2,3 \%$ & $0,3 \%$ & & \\
\hline & $11-16$ & 11 & 35 & 117 & 354 & 59 & 9 & 0 & $891,031^{1 * *}$ & 0,000 \\
\hline & & $1,9 \%$ & $6,0 \%$ & $20,0 \%$ & $60,5 \%$ & $10,1 \%$ & $1,5 \%$ & $0,0 \%$ & & \\
\hline & Total & 24 & 96 & 282 & 866 & 151 & 29 & 3 & $2711,964^{\text {*k* }}$ & 0,000 \\
\hline & & $1,7 \%$ & $6,6 \%$ & $19,4 \%$ & $59,7 \%$ & $10,4 \%$ & $2,0 \%$ & $0,2 \%$ & & \\
\hline \multirow{6}{*}{ Estado de ánimo } & $8-10$ & 10 & 23 & 59 & 443 & 308 & 23 & 0 & $1183,090^{* * *}$ & 0,000 \\
\hline & & $1,2 \%$ & $2,7 \%$ & $6,8 \%$ & $51,2 \%$ & $35,6 \%$ & $2,7 \%$ & $0,0 \%$ & & \\
\hline & $11-16$ & 10 & 20 & 47 & 299 & 202 & 7 & 0 & $778,723^{* * *}$ & 0,000 \\
\hline & & $1,7 \%$ & $3,4 \%$ & $8,0 \%$ & $51,1 \%$ & $34,5 \%$ & $1,2 \%$ & $0,0 \%$ & & \\
\hline & Total & 20 & 43 & 106 & 742 & 510 & 30 & 0 & $1960,643^{* * *}$ & 0,000 \\
\hline & & $1,4 \%$ & $3,0 \%$ & $7,3 \%$ & $51,1 \%$ & $35,1 \%$ & $2,1 \%$ & $0,0 \%$ & & \\
\hline \multirow{6}{*}{$\begin{array}{l}\text { Impresión } \\
\text { positiva }\end{array}$} & $8-10$ & 7 & 31 & 85 & 375 & 207 & 120 & 41 & $813,596^{* * *}$ & 0,000 \\
\hline & & $0,8 \%$ & $3,6 \%$ & $9,8 \%$ & $43,3 \%$ & $23,9 \%$ & $13,9 \%$ & $4,7 \%$ & & \\
\hline & $11-16$ & 2 & 10 & 59 & 255 & 143 & 77 & 39 & $569,809^{\text {***}}$ & 0,000 \\
\hline & & $0,3 \%$ & $1,7 \%$ & $10,1 \%$ & $43,6 \%$ & $24,4 \%$ & $13,2 \%$ & $6,7 \%$ & & \\
\hline & Total & 9 & 41 & 144 & 630 & 350 & 197 & 80 & $1381,356^{* \star *}$ & 0,000 \\
\hline & & $0,6 \%$ & $2,8 \%$ & $9,9 \%$ & $43,4 \%$ & $24,1 \%$ & $13,6 \%$ & $5,5 \%$ & & \\
\hline
\end{tabular}

Nota: ${ }^{* * *} p<0,001$

\section{Discusión}

Respecto a los rangos de edad estudiados, la muestra presentó una distribución normal, en la que la mayor parte de los estudiantes se ubica en la media en todas las escalas analizadas, presentando mayor puntuación en las escalas intrapersonal, adaptabilidad, estado de ánimo e impresión positiva. Desde esta perspectiva, es importante señalar que los niveles emocionales adecuados posibilitan mayor autoestima en las personas (Barling et al., 2000; Gil-Olarte et al., 2006; Herrera, Buitrago \& Perandones, 2015; Palmer et al., 2001), así como mejores procesos de adaptabilidad a la escuela (Extremera \& Fernández-Berrocal, 2003, 2004).

Se encontraron en el análisis de los percentiles diferencias estadísticamente significativas en cuanto al manejo del estrés y la adaptabilidad en función de la edad. Hubo mejores resultados en los niños de 8 a 10 años frente a los adolescentes de 11-16 años. Estos resultados se relacionan con la perspectiva respecto a que un mejor nivel emocional disminuye la ansiedad y la agresividad en la escuela (Cabello et al., 2019; Greenberg, 2003). En las demás escalas, aunque no se evidenciaron diferencias estadísticamente significativas, los niños puntuaron mejor en todos los ámbitos, a 
excepción de las escalas intrapersonal y la impresión positiva, en los cuales fueron mejores las puntuaciones de los adolescentes. Estos resultados se relacionan con la tendencia que tienen los niños de 6 a 10 años a desarrollar habilidades en cuanto al reconocimiento emocional y la empatía (Light et al., 2009).

Igualmente, los hallazgos encontrados en cuanto a las diferencias estadísticamente significativas, en las que los niños presentaron mejores condiciones que los adolescentes, pueden estar relacionadas con los planteamientos de Pastor et al. (2019) respecto a los conflictos y emociones con tendencia negativa que suelen tener los adolescentes, al igual que con los cambios biológicos y la actividad hormonal que experimentan (Greenberg \& Snell, 1997; Zimmerman \& Iwanski, 2014).

En cuanto al análisis de los niveles, omitiendo los estudiantes que se encuentran en la media, los niños presentaron mayor tendencia hacia los niveles bajos - bajo, muy bajo y marcadamente bajoen la escala intrapersonal, mientras que esta misma tendencia la tuvieron los jóvenes respecto al ámbito interpersonal, manejo del estrés, adaptabilidad y coeficiente emocional. Ahora bien, aunque los estudiantes que se ubican en estos niveles son un porcentaje reducido, es relevante recordar que estos niveles bajos implican problemas para entender las emociones y pretensiones de los demás (Dayton, 2009), además que se convierten en estudiantes que pueden presentar problemas de internalización, soledad y depresión (Davis et al., 2019).

Respecto al estado general de ánimo, y manteniendo la exclusión del grupo de estudiantes en la media, los niños evidenciaron tendencia hacia los niveles altos - alto, muy alto y marcadamente alto-, mientras que los jóvenes la presentaron en la impresión positiva. Al respecto, Bar-On y Parker (2000) señalan que es común que algunas personas tengan una impresión positiva elevada de sí mismas. En cualquier caso, es importante enfatizar que los resultados del presente estudio establecen tendencias similares a los hallazgos de otras investigaciones realizadas en Colombia (Ibáñez \& Gutiérrez, 2018; Tovar, 2018).

Para finalizar, dados los efectos positivos de los programas de educación socioemocional en el ámbito escolar (Blair et al., 2018; Coelho et al., 2015; Meyers et al., 2019; Panayiotou et al., 2019), es necesario apostar firmemente desde las instituciones educativas por 
la creación de ambientes positivos de aprendizaje fundamentados en la alfabetización emocional de niños y jóvenes (Agulló et al., 2011; Brackett et al., 2004; Cherniss et al., 2006; Eren et al., 2009; Gil-Olarte et al., 2006; Greenberg et al., 2003).

\section{Referencias}

Agulló, M., Filella, G., Soldevila, A., \& Ribes, R. (2011). Evaluación de la educación emocional en el ciclo medio de Educación Primaria. Revista de Educación, 354, 765-783. https://doi.org/10.4438/1988-592XRE-2011-354-020

Albanese, O., Stasio de, S., Chiacchio di, C., Fiorilli, C., \& Pons, F. (2010). Emotion Comprehension: The Impact of Nonverbal Intelligence. The Journal of Genetic Psychology, 171(2), 101-115. https://doi. org/10.1080/00221320903548084

Aldao, A., \& Christensen K. (2015). Linking the Expanded Process Model of Emotion Regulation to Psychopathology by focusing on behavioral outcomes of regulation. Psychological Inquiry, 26, 27-36. https://doi. org/10.1080/1047840X.2015.962399

Aldao, A., Nolen-Hoeksema, S., \& Schweizer, S. (2010). Emotionregulation strategies across psychopathology: A meta-analytic review. Clinical Psychology Review, 30, 217-237. https://doi.org/10.1016/j. cpr.2009.11.004

Armstrong, A., Galligan, R., \& Critchley, C. (2011). Emotional intelligence and psychological resilience to negative life events. Personality and Individual Differences, 51(3), 331-336. https://doi.org/10.1016/j. paid.2011.03.025

Babić, A., \& Buško, V. (2015). Emotional Intelligence in Early Adolescence: Correlates of Emotion Management Abilities and the Prediction of School Achievement. Drustvena Istrazivanja, 4(1), 21-45. https://doi. org/10.5559/di.24.1.02

Barling, J., Slater, F., \& Kelloway, E. (2000). Transformational leadership and emotional intelligence. Leadership and Organization Development Journal, 21, 157-162. https://doi.org/10.1108/01437730010325040

Bar-On, R. (1997). Bar-On Emotional Quotient Inventory: Technical Manual. Toronto: Multi Health System Inc.

Bar-On, R. (2000). Emotional and social intelligence: insights from the emotional quotient inventory. En R. Bar-On, \& J. Parker (Eds.), Handbook of emotional intelligence (pp. 363-388). San Francisco: Jossey-Bass.

Bar-On, R. (2006). The Bar-On model of Emotional-Social Intelligence (ESI). Psicothema, 18(Suppl.), 13-25. 
Bar-On, R., \& Parker, J. (2000). BarOn Emotional Quotient Inventory: Youth Version (BarOn EQi:YV), Technical Manual. Toronto: MultiHealth Systems Inc.

Bar-On, R., Tranel, D., Denburg N., \& Bechara, A. (2003). Exploring the neurological substrate of emotional and social intelligence. Brain, 126, 1790-1800. https://doi.org/10.1093/brain/awg177

Barrio del, M. (2005). Emociones infantiles. Madrid: Pirámide.

Bisquerra, R. (2009). Psicopedagogía de las emociones. Madrid: Síntesis.

Blair, C., McKinnon, R., \& Daneri, M. (2018). Effect of the tools of the mind kindergarten program on children's social and emotional development. Early Childhood Research Quarterly, 43, 52-61. https:// doi.org/10.1016/j.ecresq.2018.01.002

Boekaerts, M. (1993). Being concerned with well-being and with learning. Educational Psychologist, 28(2), 149-167. https://doi.org/10.1207/ s15326985ep2802_4

Brackett, M., Mayer, J., \& Warner, R. (2004). Emotional intelligence and its relation to everyday behavior. Personality and Individual Differences, 36, 1387-1402. https://doi.org/10.1016/So191-8869(03)o0236-8

Brackett, M., Rivers, S., Shiffman, S., Lerner, N., \& Salovey, P. (2006). Relating Emotional Abilities to Social Functioning: A Comparison of Self-Report and Performance Measures of Emotional Intelligence. Journal of Personality and Social Psychology, 91(4), 780-795. https:// doi.org/10.1037/0022-3514.91.4.780

Buitrago, R. (2012). Contexto Escolar e Inteligencia Emocional en Instituciones Educativas Públicas del Ámbito Rural y Urbano del Departamento de Boyacá (Colombia) (Tesis doctoral, Universidad de Granada, Granada, España). Recuperado de http://hera.ugr.es/ tesisugr/20956575.pdf

Buitrago, R., \& Cárdenas, R. (2017). Emociones e Identidad Profesional Docente: Relaciones e Incidencia. Praxis \& Saber, 8(17), 225-247. https://doi.org/10.19053/22160159.v8.n17.2018.7208

Buitrago, R., \& Herrera, L. (2013). Matricular las Emociones en la Escuela, una Necesidad Educativa y Social. Praxis \& Saber, 8(4), 87-108. https:// doi.org/10.19053/22160159.2653

Buitrago, R., \& Herrera, L. (2015). Coeficiente emocional en niños y niñas de $4^{\circ}$ y $5^{\circ}$ de Básica Primaria en Boyacá, Colombia. En L. Herrera, \& M. Ortiz (Coords.), Desarrollo, Educación, Diversidad y Cultura: Análisis Interdisciplinar (Líneas de Investigación del Grupo HUM-742) (pp. 205-222). Madrid: La Factoría Ediciones.

Buitrago, R., Ávila, A., \& Cárdenas, R. (2017). El Sentido y el Significado Atribuido a las Emociones por el Profesorado en Formación de 
la Universidad Pedagógica y Tecnológica de Colombia. Contextos Educativos, 20, 77-93. http://doi.org/10.18172/con.2998

Cabello, E., Pérez, N., Ros, A., \& Filella, G. (2019). Los Programas de Educación Emocional Happy 8-12 y Happy 12-16. Evaluación de su Impacto en las Emociones y el Bienestar. Revista Española de Orientación y Psicopedagogía, 3o(2), 53-66.

Charbonneau, D., \& Nicol, A. (2002). Emotional intelligence and prosocial behaviours in adolescents. Psychological Reports, 90, 361-370. https:// doi.org/10.2466/PRo.90.2.361-370

Chen, S. (2019). Chinese Adolescents' Emotional Intelligence, Perceived Social Support, and Resilience-The Impact of School Type Selection. Frontiers in Psychology, 10, 1-11. https://doi.org/10.3389/ fpsyg.2019.01299

Cherniss, C., Extein, M., Goleman, D., \& Weissberg, R. (2006). Emotional Intelligence: What Does the Research Really Indicate? Educational Psychologist, 41(4), 239-245. https://doi.org/10.1207/ S15326985ep4104_4

Ciarrochi, J., Chan, A., \& Bajgar, J. (2001). Measuring emotional intelligence in adolescents. Personality and Individual Differences, 31(7), 11051119. https://doi.org/10.1016/s0191-8869(00)00207-5

Coelho,V., Marchante, M.,\&Sousa,V.(2015). “PositiveAttitude”: Amultilevel model analysis of the effectiveness of a Social and Emotional Learning Program for Portuguese middle school students. Journal of Adolescence, 43, 29-38. http://dx.doi.org/10.1016/j.adolescence.2015.05.009

Connor, B., \& Slear, S. (2009). Emotional intelligence and anxiety; emotional intelligence and resiliency. International Journal of Learning, 16(1), 249-26o. https://doi.org/10.18848/1447-9494/CGP/v16io1/46o8

Dalai-Lama, \& Ekman, P. (2009). Sabiduría Emocional. Barcelona: Kairós.

Davis, S., Nowland, R., \& Qualter, P. (2019). The Role of Emotional Intelligence in the Maintenance of Depression Symptoms and Loneliness Among Children. Frontiers in Psychology, 10, 1-12. https:// doi.org/10.3389/fpsyg.2019.01672

Dayton, T. (2009). Equilibrio emocional. Cómo alcanzar la sobriedad emocional en la vida. Buenos Aires: Kier.

Droppert, K., Downey, L., Lomas, J., Bunnett, E., Simmons, N., Wheaton, A... Stough, C. (2019). Differentiating the contributions of emotional intelligence and resilience on adolescent male scholastic performance. Personality and Individual Differences, 145, 75-81. https://doi. org/10.1016/j.paid.2019.03.023

Eastabrook J., Flying J., \& Hollestein T. (2014). Internalizing symptoms in female adolescents: Associations with emotional awareness and 
emotion regulation. Journal of Child and Family Studies, 23, 487-496. https://doi.org/10.1007/s10826-012-9705-y

Engelberg, E., \& Sjöberg, L. (2004). Emotional intelligence, affect intensity, and social adjustment. Personality \& Individual Differences, 37(3), 533-542. https://doi.org/10.1016/j.paid.2003.09.024

Eren, E., Ergun, E., \& Altintas, O. (2009). The Relationship Between School Success and the Emotional Intelligence of Primary School Headmasters and Teachers. Journal of American Academy of Business, Cambridge, 15(1), 224-228.

Extremera, N., \& Fernández-Berrocal, P. (2003). La inteligencia emocional en el contexto educativo: hallazgos científicos de sus efectos en el aula. Revista de Educación, 332, 97-116.

Extremera, N., \& Fernández-Berrocal, P. (2004). El papel de la inteligencia emocional en el alumnado: evidencias empíricas. Revista Electrónica de Investigación Educativa, 6(2), 1-17. Recuperado de http://redie. uabc.mx/vol6no2/contenido-extremera.html.

Fernández-Berrocal, P., \& Extremera, N. (2006). Emotional intelligence and emotional reactivity and recovery in laboratory context. Psicothema, 18(Suppl.), 72-78. Recuperado de http://www.psicothema.com/ english $/$ psicothema.asp? $\mathrm{id}=3279$

Ferrándiz, C., Hernández, D., Bermejo, R., Ferrando, M., \& Sáinz, M. (2012). Social and emotional intelligence in childhood and adolescence: Spanish validation of a measurement instrument. Revista de Psicodidáctica, 17(2), 309-338. http://dx.doi.org/10.1387/Rev.Psicodidact.2814

Gerdes, K., \& Segal, E. (2011). Importance of Empathy for Social Work Practice: Integrating New Science. Social Work, 56(2), 141-148.

Gerdes, K., Segal, E., Jackson, K., \& Mullins, J. (2011). Teaching Empathy: a Framework rooted in Social Cognitive Neuroscience and Social Justice. Journal of Social Work Education, 47(1), 109-131. http://dx.doi. org/10.5175/JSWE.2011.200900085

Gil-Olarte, M., Palomera, R., \& Brackett, M. (2006). Relating emotional intelligence to social competence and academic achievement in high school students. Psicothema, 18(Suppl.), 118-123. Recuperado de http://www.psicothema.com/pdf/3286.pdf

González-Yubero, S., Palomera, R., \& Lázaro-Visa, S. (2019). Trait and Ability Emotional Intelligence as Predictors of Alcohol Consumption in Adolescents. Psicothema, 31(3), 292-297. https://doi.org/10.7334/ psicothema2018.315

Greenberg, M. (2003). La educación del corazón. En D. Goleman (Ed.), Emociones Destructivas, cómo entenderlas y superarlas (pp. 146-158). Barcelona: Editorial Kairós. 
Greenberg, M., \& Snell, J. (1997). The neurobiological basis of emotional development. En P. Salovey (Ed.), Emotional development and emotional literacy (pp. 93-119). New York, NY: Basic Books.

Greenberg, M., Weissberg, R., O’Brien, M., Zins, J., Fredericks, L., Resnik, H... Elias, M. (2003). Enhancing school-based prevention and youth development through coordinated social and emotional learning. American Psychologist, 58, 466-474. https://doi.org/10.1037/0003066X.58.6-7.466

Gross J. (1998). The emerging field of emotion regulation: An integrative review. Review of General Psychology, 2, 271-299. https://doi. org/10.1037/1089-2680.2.3.271

Gross J. (2015). Emotion regulation: Conceptual and empirical foundations. En J. Gross (Ed.), Handbook of emotion regulation (pp. 3-20). New York, NY: Guilford Press.

Gullone, E., \& Taffe, J. (2012). The Emotion Regulation Questionnaire for Children and Adolescents (ERQ-CA): A psychometric evaluation. Psychological Assessment, 24, 409-417. https://doi.org/10.1037/ a0025777

Gullone, E., Hughes, E., King, N., \& Tonge, B. (2010). The normative development of emotion regulation strategy use in children and adolescents: A 2-year follow-up study. The Journal of Child Psychology and Psychiatry, 51, 567-574. https://doi.org/10.1111/j.14697610.2009.02183.x

Gumora, G., \& Arsenio, W. (2002). Emotionally, emotion regulation, and school performance in middle school children. Journal of School Psychology, 4O(5), 395-413. http://dx.doi.org/10.1016/Soo224405(02)00108-5

Herrera, L., Buitrago, R., \& Ávila, A. (2016). Empathy in future teachers of the Pedagogical and Technological University of Colombia. New Approaches in Educational Research, 5(1), 30-37. https://doi. org/10.7821/naer.2016.1.136

Herrera, L., Buitrago, R., \& Cepero S. (2017). Emotional Intelligence in Colombian Primary School Children. Location and Gender. Universitas Psychologica, 16(3), 1-10. https://doi.org/10.11144/Javeriana.upsy163.eips

Herrera, L., Buitrago, R., \& Perandones, T. (2015). Psicología Positiva e Inteligencia Emocional en Educación. DEDiCA, Revista de Educação e Humanidades, 8, 139-153.

Herrera, L., Buitrago, R., Lorenzo, O., \& Badea, M. (2015). Socio-Emotional Intelligence in Colombian Children of Primary Education. An analysis in rural and urban settings. Procedia-Social and Behavioral Sciences, 203, 4-10. https://doi.org/10.1016/j.sbspro.2015.08.251 
Hu, T., Zhang, D., Wang, J., Mistry, R., Ran, G., \& Wang, X. (2014). Relation between emotion regulation and mental health: A meta-analysis review. Psychological Reports, 114, 341-362. https://doi.org/10.2466/03.20. PRo.114k22w4

Ibáñez, C., \& Gutiérrez, A. (2018). Relación entre Práctica Musical y Coeficiente Emocional en Niños y Adolescentes de la Ciudad de Tunja (Trabajo Fin de Grado, Universidad Pedagógica y Tecnológica de Colombia, Tunja, Colombia).

Jiménez, E., Alarcón, R., \& Vicente-Yague, M. (2019). Reading Intervention: Correlation Between Emotional Intelligence and Reading Competence in High School Students. Revista de Psicodidáctica, 24(1), 24-30. https:// doi.org/10.1016/j.psicod.2018.10.001

Kring, A., \& Sloan, D. (2010). Emotion regulation and Psychopathology: A transdiagnostic approach to etiology and treatment. New York, NY: Guilford Press.

Kun, B., Urbán, R., Paksi, B., Csóbor, L., Oláh, A., \& Demetrovics, Z. (2012). Psychometric characteristics of the Emotional Quotient Inventory, Youth Version, Short Form, in Hungarian high school students. Psychological Assessment, 24(2), 518-523. http://dx.doi.org/10.1037/a0026013

Light, S., Coan, J., Zahn-Waxler, C., Frye, C., Goldsmith, H., \& Davidson, R. (2009). Empathy Is Associated With Dynamic Change in Prefrontal Brain Electrical Activity During Positive Emotion in Children. Child Development, $80(4), 1210-1231$. https://doi.org/ 10.1111/j.14678624.2009.01326.x

Llewellyn, N., Dolcos, S., Iordan, A., Rudolph, K., \& Dolcos, F. (2013). Reappraisal and suppression mediate the contribution of regulatory focus to anxiety in healthy adults. Emotion, 13, 610-615. https://doi. org/10.1037/aoo32568

Lopes, P., Brackett, M., Nezlek, J., Schütz, A., Sellin, I., \& Salovey, P. (2004). Emotional Intelligence and Social Interaction. Personality and Social Psychology Bulletin, 30(8), 1018-1034. https://doi. org/10.1177/0146167204264762

Lopes, P., Salovey, P., Beers, M., \& Coté, S. (2005). Emotional regulation ability and the quality of social interaction. Emotion, 5(1), 113-118. https://doi. org/10.1037/1528-3542.5.1.113

Magnano, P., Craparo, G., \& Paolillo, A. (2016). Resilience and emotional intelligence: Which role in achievement motivation. International Journal of Psychological Research, 9(1), 9-20. https://doi. org/10.21500/20112084.2096

Mayer, J., Roberts, R., \& Barsade, S. (2008). Human Abilities: Emotional Intelligence. Annual Review of Psychology, 59, 507-536. https://doi. org/10.1146/annurev.psych.59.103006.093646

Mestre, J., Guil, R., Lopes, P., Salovey, P., \& Gil-Olarte, P. (2006). Emotional intelligence and social and academic adaptation to school. Psicothema, 18(Suppl.), 112-117. Recuperado de http://www.psicothema.com/ psicothema.asp?id=3285 
Meyers, D., Domitrovich, C., Dissi, R., Trejo, J., \& Greenberg, M. (2019). Supporting systemic social and emotional learning with a schoolwide implementation model. Evaluation and Program Planning, 73, 53-61. https://doi.org/10.1016/j.evalprogplan.2018.11.005

Mitrofan, N., \& Ciuluvică, C. (2012). Anger and hostility as indicators of emotion regulation and of the life satisfaction at the beginning and the ending period of the adolescence. Procedia-Social and Behavioral Sciences, 33, 65-69. https://doi.org/10.1016/j.sbspro.2012.01.084

Mozaz, M., Mestre, J., \& Núñez-Vázquez, I. (2009). Inteligencia emocional y cerebro. En J. Mestre, \& P. Fernández-Berrocal (Coords.), Manual de inteligencia emocional (pp. 123-152). Madrid: Ediciones Pirámide.

Palmer, B., Walls, M., Burgess, Z., \& Stough C. (2001). Emotional intelligence and effective leadership. Leadership \& Organization Development Journal, 22(1), 5-10. https://doi.org/10.1108/01437730110380174

Palomera, R., Fernández-Berrocal, P., \& Brackett, M. (2008). La inteligencia emocional como una competencia básica en la formación inicial de los docentes: algunas evidencias. Revista Electrónica de Investigación Psicoeducativa, 15(6), 437-454. Recuperado de http://www. investigacion-psicopedagogica.org/revista/articulos/15/espannol/ Art_15_276.pdf.

Panayiotou, M., Humphrey, N., \& Wigelsworth, M. (2019). An empirical basis for linking social and emotional learning to academic performance. Contemporary Educational Psychology, 56, 193-204. https://doi. org/10.1016/j.cedpsych.2019.01.009

Pastor, M., López-Penadés, R., Cifre, E., \& Moliner-Urdiales, D. (2019). The Spanish Version of the Emotion Regulation Questionnaire for Children and Adolescents (ERQ-CA): A Psychometric Evaluation in Early Adolescence. The Spanish Journal of Psychology, 22(e30), 1-12. https:// doi.org/10.1017/sjp.2019.30

Petrides, K., Frederickson, N., \& Furnham, A. (2004). The role of trait emotional intelligence in academic performance and deviant behavior at school. Personality and Individual Differences, 36, 277-293. https://doi. org/10.1016/So191-8869(03)00084-9

Pressman, S., \& Cohen, S. (2005). Does positive affect influence health? Psychological Bulletin, 131(6), 925-971. https://doi.org/10.1037/00332909.131.6.925

Reis, D., Brackett, M., Shamosh, N., Kiehl, K., Salovey, P., \& Gray, J. (2007). Emotional Intelligence predicts individual differences in social exchange reasoning. NeuroImage, 35, 1385-1391. https://doi.org/10.1016/j. neuroimage.2006.12.045

Roeser, R., Van der Wolf, K., \& Strobel, K. (2001). On the relation between social-emotional and school functioning during early adolescence: Preliminary findings from Dutch and American samples. Journal of School Psychology, 39, 111-139. https://doi.org/10.1016/So0224405(01)00060-7 
Saarni, C. (2000). Emotional competence: A developmental perspective. In R. Bar-On, \& D. Parker (Eds.), The Handbook of Emotional Intelligence (pp. 68-91). San Francisco: Jossey Bass.

Saracostti, M., Lara, L., Martella, D., Miranda., Miranda-Zapata, E., \& Reininger, T. (2019). Influence of Family Involvement and Children's Socioemotional Development on the Learning Outcomes of Chilean Students. Frontiers in Psychology, 1O, 1-7. https://doi.org/10.3389/ fpsyg.2019.00335

Schoeps, K., Montoya-Castilla, I., \& Raufelder, D. (2019). Does Stress Mediate the Association Between Emotional Intelligence and Life Satisfaction During Adolescence?. Journal of School Health, 89(5), 354-364. https:// doi.org/10.1111/josh.12746

Shapiro, L. (2008). La inteligencia emocional de los niños. Una guía para padres y maestros. Barcelona: Zeta.

Tovar, V. (2018). Coeficiente Emocional en Niños y Adolescentes de la Institución Educativa Marco Antonio Quijano Rico de Sogamoso Boyacá - Colombia (Tesis de Maestría, Universidad de la Amazonía, Florencia, Colombia).

Verzeletti, C., Zammuner, V., Galli, C., \& Agnoli, S. (2016). Emotion regulation strategies and psychosocial well-being in adolescence. Cogent Psychology, 3(1), 1-15. https://doi.org/10.1080/23311908.2016.1199294

Wang, L., Wei, M., Yi, E., Lo, M., \& Lee, M. (2019). The Development and Validation of the Emotional Cultivation Scale: An East Asian Cultural Perspective. Journal of Counseling Psychology, 66(4), 409-423. http:// dx.doi.org/10.1037/couooo0346

Wang, Y., Hawk, S., Tang, Y., Schlegel, K., \& Zou, H. (2019). Characteristics of Emotion Recognition Ability among Primary School Children: Relationships with Peer Status and Friendship Quality. Child Indicators Research, 12(4), 1369-1388. https://doi.org/10.1007/s12187-018-9590-Z

Webb, T., Miles, E., \& Sheeran, P. (2012). Dealing with feeling: A metaanalysis of the effectiveness of strategies derived from the process model of emotion regulation. Psychological Bulletin, 138, 775-808. https://doi. org/10.1037/a0027600

Zavala, M., Valadez, M., \& Vargas, M. (2008). Emotional intelligence and social skills in adolescents with high social acceptance. Electronic Journal of Research in Educational Psychology, 6(2), 319-338. Recuperado de http://www.investigacion-psicopedagogica.org/revista/articulos/15/ english/Art_15_271.pdf

Zimmermann, P., \& Iwanski, A. (2014). Emotion regulation from early adolescence to emerging adulthood and middle adulthood: Age differences, gender differences, and emotion-specific developmental variations. International Journal of Behavioral Development, 38, 182194. https://doi.org/10.1177/0165025413515405 